PROCEEDINGS OF THE

AMERICAN MATHEMATICAL SOCIETY

Volume 138, Number 4, April 2010, Pages 1175-1190

S 0002-9939(09)10108-9

Article electronically published on December 9, 2009

\title{
THE RESURGENCE OF IDEALS OF POINTS AND THE CONTAINMENT PROBLEM
}

\author{
CRISTIANO BOCCI AND BRIAN HARBOURNE
}

(Communicated by Bernd Ulrich)

\begin{abstract}
We relate properties of linear systems on $X$ to the question of when $I^{r}$ contains $I^{(m)}$ in the case that $I$ is the homogeneous ideal of a finite set of distinct points $p_{1}, \ldots, p_{n} \in \mathbf{P}^{2}$, where $X$ is the surface obtained by blowing up the points. We obtain complete answers for when $I^{r}$ contains $I^{(m)}$ when the points $p_{i}$ lie on a smooth conic or when the points are general and $n \leq 9$.
\end{abstract}

\section{INTRODUCTION}

Let $I$ be a homogeneous ideal in a polynomial ring $k\left[x_{0}, \ldots, x_{N}\right]=k\left[\mathbf{P}^{N}\right]=R$ over an algebraically closed field $k$ of arbitrary characteristic. Given a homogeneous ideal $I \subseteq R$, the $m$ th symbolic power of $I$ is the ideal

$$
I^{(m)}=R \cap\left(\bigcap_{P \in \operatorname{Ass}(I)}\left(I^{m} R_{P}\right)\right) .
$$

For an ideal of the kind we will mostly be interested in here, i.e., an ideal of the form $I=\bigcap_{i}\left(I\left(p_{i}\right)^{m_{i}}\right)$, where $p_{1}, \ldots, p_{n}$ are distinct points of $\mathbf{P}^{N}, I\left(p_{i}\right)$ is the ideal generated by all forms vanishing at $p_{i}$ and each $m_{i}$ is a non-negative integer, $I^{(m)}$ turns out to be $\bigcap_{i}\left(I\left(p_{i}\right)^{m m_{i}}\right)$. If $I^{m}$ is the usual power, then there is clearly a containment $I^{m} \subseteq I^{(m)}$ and indeed, for $0 \neq I \subsetneq R, I^{r} \subseteq I^{(m)}$ holds if and only if $r \geq m$ [PSC, Lemma 8.1.4]. A much more difficult problem is to determine when there are containments of the form $I^{(m)} \subseteq I^{r}$. The results of ELS and $\mathrm{HoH}$ show that $I^{(m)} \subseteq I^{r}$ holds whenever $m \geq N r$. The second author has proposed the following conjecture [PSC, Conjecture 8.4.2]:

Conjecture 1.1. Let $I \subseteq k\left[\mathbf{P}^{N}\right]$ be a homogeneous ideal. Then $I^{(m)} \subseteq I^{r}$ if $m \geq r N-(N-1)$.

This conjecture has been verified in a range of examples (such as when $I$ is the radical ideal of a finite set of generic points in $\mathbf{P}^{2}[\mathrm{BH}$ or when $I$ is a radical ideal defining a finite set of points in $\mathbf{P}^{N}$ and $r$ is a power of the characteristic when $\operatorname{char}(k)>0$ [PSC, Example 8.4.4] or when $I$ is a monomial ideal PSC, Example 8.4.5]). Even if this conjecture is true, there is still the question of determining

Received by the editors March 14, 2008, and, in revised form, May 26, 2009.

2010 Mathematics Subject Classification. Primary 14C20, 13C05; Secondary 14N05, 14H20, $41 \mathrm{~A} 05$.

Key words and phrases. Fat points, symbolic powers, normal generation, projective space.

This research was partially supported by GNSAGA of INdAM (Italy) and by the NSA. 
for any given ideal $I$ and each $r$ what the least $m$ is for which $I^{(m)} \subseteq I^{r}$ holds. An asymptotic version of this problem is to determine the least real number $\rho(I)$, called the resurgence of $I[\mathrm{BH}]$, such that $m>r \rho(I)$ implies $I^{(m)} \subseteq I^{r}$. Thus the result of [ELS and $[\mathrm{HoH}]$ shows that $\rho(I) \leq N$. This is optimal in the sense that for any real number $c<N,[\mathrm{BH}]$ constructs an ideal $I$ with $\rho(I)>c$.

In this paper we will address in a range of cases both the problem of computing the resurgence and the problem of finding all $m$ and $r$ such that containment holds, for ideals defining fat point subschemes of $\mathbf{P}^{2}$, whose general definition we now recall. Given distinct points $p_{i} \in \mathbf{P}^{N}$ and non-negative integers $m_{i}$, we denote by $Z=m_{1} p_{1}+\cdots+m_{n} p_{n} \subset \mathbf{P}^{N}$ the subscheme (known as a fat point subscheme) defined by $I(Z)=\bigcap_{i} I\left(p_{i}\right)^{m_{i}}$, where $I\left(p_{i}\right)$ is the ideal generated by all forms which vanish at $p_{i}$.

We also recall various additional definitions we will need. Given any homogeneous ideal $(0) \neq I \subsetneq k\left[\mathbf{P}^{N}\right]$, following $[\mathrm{BH}]$, we use the following notation:

- $\alpha(I)$ is the degree of a homogeneous generator of $I$ of least degree (equivalently, it is the $M$-adic order of $I$, i.e., the largest $t$ such that $M^{t}$ contains $I$, where $M$ is the maximal homogeneous ideal of $k\left[\mathbf{P}^{N}\right]$ ),

- $\gamma(I)=\lim _{m \rightarrow \infty} \alpha\left(I^{(m)}\right) / m$,

- $\rho(I)$ is the supremum of all ratios $m / r$ such that $I^{(m)} \nsubseteq I^{r}$,

- $\operatorname{reg}(I)$ is the Castelnuovo-Mumford regularity; if $I$ is the ideal of a fat point subscheme, it is the least degree $t>0$ such that $\operatorname{dim}(R / I)_{t}=\operatorname{dim}(R / I)_{t-1}$, where $\operatorname{dim}(R / I)_{i}=\operatorname{dim} R_{i}-\operatorname{dim} I_{i}$ and $I_{i}$ (resp. $\left.R_{i}\right)$ is the vector space span in $I$ (resp. $R$ ) of the forms of degree $i$ in $I$ (resp. $R$ ).

The quantities above are related. We note that by $[\mathrm{BH}]$ we have $\rho(I) \geq \alpha(I) / \gamma(I)$ $\geq \alpha\left(I^{m}\right) / \alpha\left(I^{(m)}\right)$ for all $m \geq 1$. (Note that $\gamma(I) \geq 1$ since $I \neq k\left[\mathbf{P}^{N}\right]$, by [PSC, Lemma 8.2.2]. For example, if $Z \neq 0$ is a fat point subscheme, then $Z$ contains some point $p$ as a subscheme. Thus $\alpha(I(m p)) \leq \alpha(I(m Z))$, so using the easy fact that $m=\alpha(I(m p))$ we see that $\gamma(I(Z)) \geq 1$. It is also true that $\rho(I(Z)) \geq 1$ when $Z \neq 0$. To see this, note that $\alpha(I(m Z)) \leq m \alpha(I(Z))$ for all $m \geq 1$, so $1 \leq \alpha(I(Z)) / \gamma(I(Z))$; now use $\alpha(I(Z)) / \gamma(I(Z)) \leq \rho(I(Z))$ [BH].) Of course, $\alpha\left(I^{m}\right) / \alpha\left(I^{(m)}\right)$ gives a measure of the growth that occurs when an ordinary power $I^{m}$ is replaced by a symbolic power $I^{(m)}$, but it is possible that $\alpha\left(I^{m}\right) / \alpha\left(I^{(m)}\right)=1$ even though $\rho(I)>1$ and $I^{m} \subsetneq I^{(m)}$ for all $m>1$ (see Lemma 3.1 and Theorem 3.4). Thus $\rho(I)$ gives an asymptotic measure of additional growth not detected by $\alpha\left(I^{m}\right) / \alpha\left(I^{(m)}\right)$.

An additional important relationship was found in $[\mathrm{BH}]$. If $I \subset k\left[\mathbf{P}^{N}\right]$ is the ideal of a 0 -dimensional subscheme, then $\alpha(I) / \gamma(I) \leq \rho(I) \leq \operatorname{reg}(I) / \gamma(I)$, and hence when $\alpha(I)=\operatorname{reg}(I)$, we have an exact determination $\rho(I)=\alpha(I) / \gamma(I)$. In this situation, as an immediate consequence of [BH, Lemmas 2.3.2(a) and 2.3.4], we also have the following solution for the containment problem:

Corollary 1.2. Assume $I \subset k\left[\mathbf{P}^{N}\right]$ is the ideal of a 0 -dimensional subscheme of $\mathbf{P}^{N}$, and that $\alpha(I)=\operatorname{reg}(I)$. Then $I^{(m)} \subseteq I^{r}$ if and only if $\alpha\left(I^{(m)}\right) \geq r \alpha(I)$.

Unfortunately, $\alpha(I)=\operatorname{reg}(I)$ often does not hold, and even when it does it can be very hard to compute $\alpha\left(I^{(m)}\right)$ when $m$ is large. For example, say $I$ is the ideal defining $n$ generic points in $\mathbf{P}^{2}$. Then the value of $\gamma(I)$ is not in general known for $n>9$ (it is conjectured to be $\gamma(I)=\sqrt{n}[\overline{\mathrm{BH}}$, Section 1.3] when $n>9$ ) nor is 
$\alpha\left(I^{(m)}\right)$ in general known for $n>9$ (by the SHGH Conjecture of Segre-HarbourneGimigliano-Hirschowitz [S, [H4, G, Hi], it is conjectured that $\alpha\left(I^{(m)}\right)$ is the least $t$ such that $\left(\begin{array}{c}t+2 \\ 2\end{array}\right)>n\left(\begin{array}{c}m+1 \\ 2\end{array}\right)$ when $\left.n>9\right)$. For $n \leq 9$ generic points in $\mathbf{P}^{2}, \alpha\left(I^{(m)}\right)$ has been known for a long time [C], and one can also compute $\gamma(I)$ in these cases. When $n$ is a square, $\gamma(I)$ is known [BH, Section 1.3], and recent work has also determined $\alpha\left(I^{(m)}\right)$ when $n$ is a square $\mathrm{CM}, \mathrm{E}, \mathbf{R}$, verifying the SHGH Conjecture.

On the other hand, it is easy to specify when $\alpha(I)=\operatorname{reg}(I)$ for the ideal $I$ of $n$ generic points of $\mathbf{P}^{2}$ : this occurs exactly when $n=\left(\begin{array}{c}s+1 \\ 2\end{array}\right)$ for some $s \geq 1$, in which case $\alpha(I)=\operatorname{reg}(I)=s$. Thus Corollary 1.2 gives a complete explicit solution (i.e., $\rho(I)=\alpha(I) / \gamma(I)$ and $I^{(m)} \subseteq I^{r}$ if and only if $\left.\alpha\left(I^{(m)}\right) \geq r \alpha(I)\right)$ to the containment problem for the ideal $I$ of $n$ generic points in those cases for which $\alpha(I)=\operatorname{reg}(I)$ such that $\gamma(I)$ is known and $\alpha\left(I^{(m)}\right)$ is known for all $m \geq 1$, i.e., when $n$ is either $1,3,6$ or $n$ is any square which is at the same time a binomial coefficient. (This happens infinitely often, starting with $s=1,8,49,288, \ldots[\mathrm{BH}$, Section 1.3].)

Here we obtain results for ideals $I$ defining points $p_{1}, \ldots, p_{n} \in \mathbf{P}^{2}$ in a range of cases for which $\alpha(I) \neq \operatorname{reg}(I)$, by applying geometrically relevant properties of linear systems on the variety $X$ obtained by blowing up the points $p_{i}$. Indeed, we completely determine the set of ordered pairs $(m, r)$ for which $I^{(m)} \subseteq I^{r}$ holds in case $I \subset k\left[\mathbf{P}^{2}\right]$ is the ideal of a finite set $p_{1}, \ldots, p_{n} \in \mathbf{P}^{2}$ of points when either the points are general and $n \leq 9$ or the points lie on an irreducible conic in $\mathbf{P}^{2}$ for any $n$. At the same time we determine $\rho(I)$ in these cases.

\section{BACKGROUND}

We now recall or prove results we will need later.

In this section, let $\pi: X \rightarrow \mathbf{P}^{2}$ be obtained by blowing up distinct points $p_{1}, \ldots, p_{n} \in \mathbf{P}^{2}$. Let $L$ be the pullback via $\pi$ to $X$ of a general line in $\mathbf{P}^{2}$ and let $E_{i}$ be the blowup of $p_{i}$. Given the fat point subscheme $Z=m_{1} p_{1}+\cdots+m_{n} p_{n}$, the ideal $I(Z)$ is homogeneous. Let $I(Z)_{t}$ be the homogeneous component of $I(Z)$ of degree $t$; i.e., $I(Z)_{t}$ is the $k$-vector space span of the forms in $I(Z)$ of degree $t$. Then we have a natural identification of $I(Z)_{t}$ with $H^{0}\left(X, \mathcal{O}_{X}\left(F_{t}(Z)\right)\right)$, where $F_{t}(Z)$ denotes $t L-m_{1} E_{1}-\cdots-m_{n} E_{n}$ (see, for example, [H7, Proposition IV.1.1]).

The linear equivalence classes of the divisors $L, E_{1}, \ldots, E_{n}$ give an orthogonal basis for the divisor class group $\mathrm{Cl}(X)$ of $X$ such that $-L^{2}=E_{i}^{2}=-1$. With respect to this basis, the canonical class $K_{X}$ is $K_{X}=\left[-3 L+E_{1}+\cdots+E_{n}\right]$. For simplicity we will suppress the brackets when writing the class $\left[a L-m_{1} E_{1}-\cdots-\right.$ $\left.m_{n} E_{n}\right]$ of a divisor $a L-m_{1} E_{1}-\cdots-m_{n} E_{n}$; in context the meaning will always be clear. Also, given a divisor $F$, when indicating cohomology, we will for example often write $H^{0}(X, F)$ in place of $H^{0}\left(X, \mathcal{O}_{X}(F)\right)$.

We will say that a divisor $F$ is normally generated if the natural map

$$
H^{0}(X, F)^{\otimes s} \rightarrow H^{0}(X, s F)
$$

is surjective for all $s \geq 1$. Note that we do not require that $F$ be ample. Thus, for example, $L-E_{1}$ is normally generated and nef but not ample. (We recall that a divisor or divisor class $D$ is nef if $D \cdot C \geq 0$ for every effective divisor $C$, and $D$ is ample if $D^{2}>0$ and $D \cdot C>0$ for every effective divisor $C$ [ $\mathrm{Hr}$. Theorem V.1.10].)

Our hypothesis in this section that $Q=2 L-E_{1}-\cdots-E_{n}$ is the class of an effective divisor simply means that the points $p_{i}$ lie on a conic. Thus the results of this section are useful for analyzing the case of points on conics, where we can hope 
to get complete answers. However, when the conic is not irreducible (equivalently, not smooth), this analysis requires a treatment of subcases (depending on how many points are on each of the two lines of which the conic is composed). Our main interest in this paper is the case of $n \leq 9$ general points. Since any $n \leq 5$ general points lie on a smooth conic, we will in the next section apply the results of this section for points on a smooth conic (and obtain for free a complete answer for any number of points on a smooth conic). However, the results of this section do not require that the conic be smooth, so with a view to using the results below to analyze the case of points on reducible conics in the future, we state our results here assuming only that $Q=2 L-E_{1}-\cdots-E_{n}$ is the class of an effective divisor.

Lemma 2.1. Assume $Q=2 L-E_{1}-\cdots-E_{n}$ is the class of an effective divisor on $X$ (i.e., the points $p_{i}$ lie on a plane conic, not necessarily smooth). If $F$ is nef, then the class of $F$ is the class of an effective divisor, $h^{1}(X, F)=0,|F|$ is base point free and $F$ is normally generated.

Proof. If $F$ is nef, then $F \cdot L \geq 0$ since $L$ is effective (so $\left(K_{X}-F\right) \cdot L \leq K_{X} \cdot L=-3$, and thus $h^{2}(X, F)=h^{0}\left(X, K_{X}-F\right)=0$ since $L$ is also nef) and $F^{2} \geq 0$ [H5, Proposition II.3], but $-K_{X}=Q+L$ is effective, so $-F \cdot K_{X} \geq 0$; hence by Riemann-Roch, $h^{0}(X, F) \geq\left(F^{2}-F \cdot K_{X}\right) / 2+1>0$, so $F$ is effective. Moreover, $h^{1}(X, F)=0$ and $|F|$ is base point free by Lemma 3.1.1(b) of [H2]. If in addition $F^{2}>0$ and $-K_{X} \cdot F \geq 3$, then $F$ is normally generated by Proposition 3.1 of [H1]. But $-K_{X}=Q+L$, so $F$ nef means $-K_{X} \cdot F \geq L \cdot F \geq 0$. If $L \cdot F=0$, then it is easy to see that $F=0$, which trivially is normally generated. If $L \cdot F=1$, then either $F=L$ or $F=L-E_{i}$ for some $i$, and it is easy to see that in both cases $F$ is normally generated. Finally, if $L \cdot F=2$, then up to reordering the points $p_{i}, F$ must be either $2 L, 2 L-E_{1}, \ldots, 2 L-E_{1}-\cdots-E_{4}$ or $2 L-2 E_{1}$ (since if $m_{i}>2$ for some $i$, then $F \cdot\left(L-E_{i}\right)<0$, but $L-E_{i}$ is nef, and if $F=2 L-E_{1}-\cdots-E_{i}$ for $i>4$, then $F^{2}<0$; while if $F=2 L-m_{1} E_{1}-\cdots-m_{i} E_{i}$ where $m_{1}>1$ and $m_{2}>0$, then $F \cdot\left(L-E_{1}-E_{2}\right)<0$, which contradicts the assumption that $F$ is nef since $L-E_{1}-E_{2}$ is linearly equivalent to an effective divisor). In each case $F$ nef implies $-K_{X} \cdot F \geq 3$ and $F^{2}>0$ unless either $F=2\left(L-E_{i}\right)$ or $F=2 L-E_{i_{1}}-\cdots-E_{i_{4}}$, but in both cases $F$ is normally generated, since a single point and also four points on an irreducible conic are complete intersections; and hence in these cases each element of $|m F|$ is a sum of $m$ components, each component being an element of $|F|$ (i.e., the map $H^{0}(X, F)^{\otimes m} \rightarrow H^{0}(X, m F)$ is onto).

Lemma 2.2. Assume $Q=2 L-E_{1}-\cdots-E_{n}$ is the class of an effective divisor on $X$. If $F$ is nef, then $H^{0}(X, F) \otimes H^{0}(X, L) \rightarrow H^{0}(X, F+L)$ is surjective.

Proof. This is Theorem 3.1.2 of [H2]. See also Lemma 2.11 of [GuH].

If $F$ and $G$ are nef, it is not always true that $H^{0}(X, F) \otimes H^{0}(X, G) \rightarrow H^{0}(X, F+$ $G$ ) is surjective, even if $Q=2 L-E_{1}-\cdots-E_{n}$ is the class of an effective divisor. The next two results give special cases where surjectivity does hold more generally than what we have by Lemma 2.2

Corollary 2.3. Assume $Q=2 L-E_{1}-\cdots-E_{n}$ is the class of an effective divisor on $X$. Let $i, j, r, s$ be non-negative integers. If $F$ is nef, then $H^{0}(X, i F+j L) \otimes$ $H^{0}(X, r F+s L) \rightarrow H^{0}(X,(i+r) F+(j+s) L)$ is surjective. 
Proof. By Lemma 2.1 $H^{0}(X, F)^{\otimes i+r} \rightarrow H^{0}(X,(i+r) F)$ is surjective and thus induces a surjective map

$$
H^{0}(X, F)^{\otimes i+r} \otimes H^{0}(X, L)^{\otimes j+s} \rightarrow H^{0}(X,(i+r) F) \otimes H^{0}(X, L)^{\otimes j+s},
$$

and by Lemma $2.2 H^{0}(X,(i+r) F) \otimes H^{0}(X, L)^{\otimes j+s} \rightarrow H^{0}(X,(i+r) F+(j+s) L)$ is surjective; hence $H^{0}(X, F)^{\otimes i+r} \otimes H^{0}(X, L)^{\otimes j+s} \rightarrow H^{0}(X,(i+r) F+(j+s) L)$ is surjective. But this map factors as

$$
\begin{aligned}
& H^{0}(X, F)^{\otimes i+r} \otimes H^{0}(X, L)^{\otimes j+s} \\
& \rightarrow H^{0}(X, i F) \otimes H^{0}(X, j L) \otimes H^{0}(X, r F) \otimes H^{0}(X, s L) \\
& \rightarrow H^{0}(X, i F+j L) \otimes H^{0}(X, r F+s L) \rightarrow H^{0}(X,(i+r) F+(j+s) L) ;
\end{aligned}
$$

hence $H^{0}(X, i F+j L) \otimes H^{0}(X, r F+s L) \rightarrow H^{0}(X,(i+r) F+(j+s) L)$ is surjective.

Let us say that a divisor class $F=i L-m_{1} E_{1}-\cdots-m_{n} E_{n}$ is uniform (some authors use the term homogeneous here) if $m_{1}=\cdots=m_{n}=m$ for some $m$. Likewise we say that $Z=m_{1} p_{1}+\cdots+m_{n} p_{n}$ is uniform if $m_{1}=\cdots=m_{n}=m$ for some $m$.

Proposition 2.4. Assume $Q=2 L-E_{1}-\cdots-E_{n}$ is the class of an effective divisor on $X$. If $F$ is nef and if $G$ is nef and uniform, then $H^{0}(X, F) \otimes H^{0}(X, G) \rightarrow$ $H^{0}(X, F+G)$ is surjective.

Proof. If $n=0$, then $X=\mathbf{P}^{2}$, so a nef divisor must be of the form $i L$ for $i \geq 0$, while if $n=1$, a nef divisor is of the form $i\left(L-E_{1}\right)+j L$ for non-negative $i$ and $j$. Thus for $n \leq 1$ the result follows by Corollary 2.3. Also, if $G=i L$, then again the result follows by Corollary 2.3. Thus we may assume that $G=i L-m\left(E_{1}+\cdots+E_{n}\right)$ with $m>0$ and $n \geq 2$. Note that $G-Q$ is nef (and uniform) in this case (and hence $h^{1}\left(X, \mathcal{O}_{X}(G-Q)\right)=0$ by Lemma 2.1): to see that $G-Q$ is nef, note that since $G$ is nef, we have $G \cdot\left(L-E_{1}-E_{2}\right) \geq 0$, so $i \geq 2 m$. Thus $G=m Q+(i-2 m) L$, so $G-Q$ is effective and nefness follows if $(G-Q) \cdot D \geq 0$ for every irreducible component $D$ of $C$, where $C$ is an effective divisor whose class is $Q$. If in fact $D \cdot(G-Q)<0$, then $0>D \cdot(G-Q)=D \cdot((i-2 m) L+(m-1) Q)$ implies $D \cdot Q<0$; hence $0>D \cdot(G-Q) \geq D \cdot G$. Since $G$ is nef, $D \cdot G<0$ is impossible; hence $D \cdot(G-Q)<0$ is also impossible, so $G-Q$ is nef.

Now consider the exact sequence $0 \rightarrow \mathcal{O}_{X}(F-Q) \rightarrow \mathcal{O}_{X}(F) \rightarrow \mathcal{O}_{Q}(F) \rightarrow 0$. Since $-K_{X}=Q+L$ we have $h^{1}\left(X, \mathcal{O}_{X}(F-Q)\right)=h^{1}\left(X, \mathcal{O}_{X}\left(F+L+K_{X}\right)\right)$, and by Serre duality this becomes $h^{1}\left(X, \mathcal{O}_{X}(-(F+L))\right)$; and since $F+L$ is nef and big, $h^{1}\left(X, \mathcal{O}_{X}(-(F+L))\right)=0$ by Ramanujam vanishing (for the characteristic $p$ version, see Theorem 1.6 [T] or Theorem 2.8 of [H1] $)$. Thus $H^{0}\left(X, \mathcal{O}_{X}(F)\right) \rightarrow$ $H^{0}\left(Q, \mathcal{O}_{Q}(F)\right)$ is surjective.

Consider the maps

$$
\begin{gathered}
\mu_{1}: H^{0}\left(X, \mathcal{O}_{X}(G-Q)\right) \otimes H^{0}\left(X, \mathcal{O}_{X}(F)\right) \rightarrow H^{0}\left(X, \mathcal{O}_{X}(F+G-Q)\right), \\
\mu_{2}: H^{0}\left(X, \mathcal{O}_{X}(G)\right) \otimes H^{0}\left(X, \mathcal{O}_{X}(F)\right) \rightarrow H^{0}\left(X, \mathcal{O}_{X}(F+G)\right),
\end{gathered}
$$

and

$$
\mu_{3}: H^{0}\left(Q, \mathcal{O}_{Q}(G)\right) \otimes H^{0}\left(X, \mathcal{O}_{X}(F)\right) \rightarrow H^{0}\left(Q, \mathcal{O}_{Q}(F+G)\right) .
$$

Taking global sections of $0 \rightarrow \mathcal{O}_{X}(G-Q) \rightarrow \mathcal{O}_{X}(G) \rightarrow \mathcal{O}_{Q}(G) \rightarrow 0$ we get a short exact sequence; tensor by $H^{0}\left(X, \mathcal{O}_{X}(F)\right)$ and map to the short exact sequence given 
by taking global sections of $0 \rightarrow \mathcal{O}_{X}(F+G-Q) \rightarrow \mathcal{O}_{X}(F+G) \rightarrow \mathcal{O}_{Q}(F+G) \rightarrow 0$ to obtain the following diagram (where $V_{F}$ denotes $H^{0}\left(X, \mathcal{O}_{X}(F)\right)$ ):

$$
\begin{gathered}
0 \rightarrow H^{0}\left(X, \mathcal{O}_{X}(G-Q)\right) \otimes V_{F} \rightarrow H^{0}\left(X, \mathcal{O}_{X}(G)\right) \otimes V_{F} \rightarrow H^{0}\left(Q, \mathcal{O}_{Q}(G)\right) \otimes V_{F} \rightarrow 0 \\
\downarrow^{\mu_{1}} \quad \downarrow^{\mu_{2}} \\
\left.0 \rightarrow H^{0}\left(X, \mathcal{O}_{X}(F+G-Q)\right) \rightarrow H^{0}\left(X, \mathcal{O}_{X}(F+G)\right) \rightarrow H^{0}\left(Q, \mathcal{O}_{Q}(F+G)\right)\right) \rightarrow 0 .
\end{gathered}
$$

By the snake lemma we have an exact sequence cok $\mu_{1} \rightarrow \operatorname{cok} \mu_{2} \rightarrow \operatorname{cok} \mu_{3} \rightarrow 0$. Since $H^{0}\left(X, \mathcal{O}_{X}(F)\right) \rightarrow H^{0}\left(Q, \mathcal{O}_{Q}(F)\right)$ is surjective, $\mu_{3}$ and $\mu_{4}: H^{0}\left(Q, \mathcal{O}_{Q}(G)\right) \otimes$ $H^{0}\left(Q, \mathcal{O}_{Q}(F)\right) \rightarrow H^{0}\left(Q, \mathcal{O}_{Q}(F+G)\right)$ have the same cokernel. We thus have an exact sequence cok $\mu_{1} \rightarrow \operatorname{cok} \mu_{2} \rightarrow \operatorname{cok} \mu_{4} \rightarrow 0$. By Lemma 2.11 of [GuH], $\operatorname{cok} \mu_{4}=0$. Thus cok $\mu_{2}=0$ if $\operatorname{cok} \mu_{1}=0$, and this last follows by induction on $m$, using the fact that $G-Q$ is nef and uniform, eventually reducing to the case $G=i L$ done above.

The notion of nefness in the context of the preceding results can be described algebraically, denoting by $\omega(I)$ the largest degree among elements of any minimal set of homogeneous generators of $I$.

Corollary 2.5. Assume $2 L-E_{1}-\cdots-E_{n}$ is the class of an effective divisor, and let $0 \neq Z=m_{1} p_{1}+\cdots+m_{n} p_{n}$ be a fat point subscheme. Then $F_{t}(Z)$ is nef if and only if $t \geq \omega(I(Z))$.

Proof. First suppose $F_{t}(Z)$ is not nef; hence there is an effective prime divisor $C$ with $F_{t}(Z) \cdot C<0$. If $F_{t}(Z)$ is not the class of an effective divisor, then $I(Z)_{t}=(0)$; hence $t<\alpha(I) \leq \omega(I)$. If $F_{t}(Z)$ is the class of an effective divisor, then $C$ is a component of every element of $\left|F_{t}(Z)\right|$; i.e., $C$ is a fixed component of $\left|F_{t}(Z)\right|$, and hence the zero-locus of $I(Z)_{t}$ is 1-dimensional, so $I(Z)$ requires a generator in some degree bigger than $t$, and again we have $t<\omega(I)$. Conversely, by Lemma 2.2 we see that if $F_{t}(Z)$ is nef, then $t \geq \omega(I(Z))$.

\section{Points ON SMOOTH CONICS}

We now focus on the case of points on a smooth plane conic. We will use the following notation. Given $Z=p_{1}+\cdots+p_{n}$ contained in a smooth plane conic $Q^{\prime}$ defined by a form $f$, and given any vector space $V$ of forms of equal degree, let $q(V)$ denote the largest exponent $e$ such that $f^{e}$ is a factor of every element of $V$.

Lemma 3.1. For $n \geq 5$, assume the points $p_{1}, \ldots, p_{n}$ lie on a smooth conic curve $Q^{\prime}$ defined by a degree 2 form $f$; hence $Q=2 L-E_{1}-\cdots-E_{n}$ is the class of a smooth curve with $Q^{2}<0$. Let $I=I(m Z)$, where $Z=p_{1}+\cdots+p_{n}$. Then $\alpha\left(I^{r}\right)=2 m r$, and, for each $t \geq 2 m r, I^{r}{ }_{t}=f^{q} I((r m-q) Z)_{t-2 q}$, where $q=q\left(I_{t}^{r}\right)$ is the minimum value of $q\left(I_{t_{1}}\right)+\cdots+q\left(I_{t_{r}}\right)$ over all sums $t=t_{1}+\cdots+t_{r}$ with $t_{i} \geq 2 m$ for all $i$, and $q\left(I_{t_{i}}\right)$ is the least $s \geq 0$ such that $2\left(t_{i}-2 s\right) \geq(m-s) n$.

Proof. Since $H=2 L-E_{1}-\cdots-E_{4}$ is easily seen to be the class of a reduced irreducible divisor of self-intersection 0 , it is nef, but $H \cdot\left((2 m-1) L-m E_{1}-\right.$ $\left.\cdots-m E_{n}\right)<0$, so $\alpha(I) \geq 2 m$. Since clearly $f^{m} \in I$, we see $\alpha(I)=2 m$; hence $\alpha\left(I^{r}\right)=r \alpha(I)=2 m r$. Since $f^{m} \in I_{2 m}$ and $I_{2 m}$ can be identified with $H^{0}(X, m Q)$, and since $m Q$ is a multiple of a prime divisor of negative self-intersection, we see that $h^{0}(X, m Q)=1$ and thus $f^{m}$ spans $I_{2 m}$. But the greatest common factor of $I_{t}$ divides that of $I_{2 m}$ for any $t \geq 2 m$, so for any $t \geq 2 m$, the greatest common factor of $I_{t}$ is a power of $f$ and hence defines a divisor $q\left(I_{t}\right) Q^{\prime}$. Let $q^{\prime}=q\left(I_{t}\right)$. 
Dividing out by this gcd leaves $I\left(\left(m-q^{\prime}\right) Z\right)_{t-2 q^{\prime}}$, which is fixed component free. Thus $F_{t-2 q^{\prime}}\left(\left(m-q^{\prime}\right) Z\right)$ is nef and uniform. In particular, $F_{t-2 q^{\prime}}\left(\left(m-q^{\prime}\right) Z\right) \cdot Q \geq 0$, so $2\left(t-2 q^{\prime}\right)-\left(m-q^{\prime}\right) n \geq 0$. Let $F=F_{t-2\left(q^{\prime}-1\right)}\left(\left(m-\left(q^{\prime}-1\right)\right) Z\right)$. Since $Q^{\prime}$ is a common divisor for $I\left(\left(m-\left(q^{\prime}-1\right)\right) Z\right)_{t-2\left(q^{\prime}-1\right)},|F|$ is not fixed component free; hence $F$ is not nef by Lemma 2.1. Since $F=\left(t-2\left(m-\left(q^{\prime}-1\right)\right) L+\left(m-\left(q^{\prime}-1\right)\right) Q\right.$, the only prime divisor which $F$ could meet negatively is $Q$; hence $0>F \cdot Q=$ $2\left(t-2\left(q^{\prime}-1\right)\right)-\left(m-\left(q^{\prime}-1\right)\right) n$. Thus whenever $t \geq 2 m, q\left(I_{t}\right)$ is the least $s \geq 0$ such that $2(t-2 s) \geq(m-s) n$.

If $t \geq \alpha\left(I^{r}\right)$, by the definition of powers of an ideal (and the fact that $I_{t}=0$ for $t<\alpha(I)=2 m$ ) we have $I_{t}^{r}=\sum_{\mathbf{j} \in S} \prod_{i=1}^{r} I_{j_{i}}$, where $S$ is the set of all sequences $\mathbf{j}=\left\{j_{1}, \ldots, j_{r}\right\}$, such that $2 m \leq j_{1} \leq \cdots \leq j_{r}$ with $j_{1}+\cdots+j_{r}=t$ (we ignore sequences with $j_{1}<2 m$ since $I_{j_{1}}=0$ for $j_{1}<2 m$ ). Thus $q$ is the minimum $q\left(\prod_{i=1}^{r} I_{j_{i}}\right)$ among all $\mathbf{j} \in S$. Of course, for $\mathbf{j}^{\prime} \in S$, we have $q\left(\prod_{i=1}^{r} I_{j_{i}^{\prime}}\right)=\sum_{i} q\left(I_{j_{i}^{\prime}}\right)$. Let $q_{i}^{\prime}=q\left(I_{j_{i}^{\prime}}\right)$ and, recycling notation, let $q^{\prime}=\sum_{i} q_{i}^{\prime}$. Then

$$
\begin{aligned}
\prod_{i=1}^{r} I_{j_{i}^{\prime}}=\prod_{i=1}^{r} f^{q_{i}^{\prime}} I\left(\left(m-q_{i}^{\prime}\right) Z\right)_{j_{i}^{\prime}-2 q_{i}^{\prime}} & =f^{q^{\prime}} \prod_{i=1}^{r} I\left(\left(m-q_{i}^{\prime}\right) Z\right)_{j_{i}^{\prime}-2 q_{i}^{\prime}} \\
& \subseteq f^{q^{\prime}} I\left(\sum_{i}\left(m-q_{i}^{\prime}\right) Z\right)_{t-2 q^{\prime}} \\
& =f^{q^{\prime}} I\left(\left(r m-q^{\prime}\right) Z\right)_{t-2 q^{\prime}} .
\end{aligned}
$$

We can identify $\prod_{i=1}^{r} I\left(\left(m-q_{i}^{\prime}\right) Z\right)_{j_{i}^{\prime}-2 q_{i}^{\prime}}$ inside $I\left(\left(r m-q^{\prime}\right) Z\right)_{t-2 q^{\prime}}$ with the image of

$$
\begin{aligned}
\bigotimes_{i=1}^{r} H^{0}\left(X, \mathcal{O}_{X}\left(F_{j_{i}^{\prime}-2 q_{i}^{\prime}}\left(\left(m-q_{i}^{\prime}\right) Z\right)\right)\right) & \rightarrow H^{0}\left(X, \mathcal{O}_{X}\left(\sum_{i=1}^{r} F_{j_{i}^{\prime}-2 q_{i}^{\prime}}\left(\left(m-q_{i}^{\prime}\right) Z\right)\right)\right) \\
& =H^{0}\left(X, \mathcal{O}_{X}\left(F_{t-2 q^{\prime}}\left(\left(r m-q^{\prime}\right) Z\right)\right)\right) .
\end{aligned}
$$

By Proposition [2.4, this map is surjective, and hence the inclusion $\prod_{i=1}^{r} I_{j_{i}^{\prime}} \subseteq$ $f^{q^{\prime}} I\left(\left(r m-q^{\prime}\right) Z\right)_{t-2 q^{\prime}}$ above is an equality. Since $q \leq q^{\prime}$, we thus see for every $\mathbf{j}^{\prime} \in S$ that $\prod_{i=1}^{r} I_{j_{i}^{\prime}}=f^{q} f^{q^{\prime}-q} I\left(\left(r m-q^{\prime}\right) Z\right)_{t-2 q^{\prime}}$ is contained in $\prod_{i=1}^{r} I_{j_{i}}=f^{q} I((r m-$ q) $Z)_{t-2 q}$ for that $\mathbf{j}=\left\{j_{1}, \ldots, j_{r}\right\}$ giving the minimum value $q$, and hence

$$
f^{q} I((r m-q) Z)_{t-2 q}=\prod_{i=1}^{r} I_{j_{i}} \subseteq I_{t}^{r}=\sum_{\mathbf{j}^{\prime} \in S} \prod_{i=1}^{r} I_{j_{i}^{\prime}} \subseteq f^{q} I((r m-q) Z)_{t-2 q} .
$$

Corollary 3.2. Let $n \geq 5$ and assume the points $p_{1}, \ldots, p_{n}$ lie on a smooth conic curve $Q^{\prime}$ defined by a degree 2 form $f$. Let $I=I(s Z)$ for $Z=p_{1}+\cdots+p_{n}$ and $s>0$. Let $m \geq r$ and let $t \geq 2 m s$. Then $I_{t}^{(m)} \subseteq I_{t}^{r}$ if and only if $q\left(I_{t}^{r}\right) \leq q\left(I_{t}^{(m)}\right)$.

Proof. Let $q_{1}=q\left(I^{r}\right)$ and $q_{2}=q\left(I_{t}^{(m)}\right)$. Suppose we have $I_{t}^{(m)} \subseteq I_{t}^{r}$; by Lemma 3.1 we have

$$
f^{q_{2}} I\left(\left(m s-q_{2}\right) Z\right)_{t-2 q_{2}}=I_{t}^{(m)} \subseteq I_{t}^{r}=f^{q_{1}} I\left(\left(r s-q_{1}\right) Z\right)_{t-2 q_{1}},
$$

which implies that $f^{q_{1}}$ divides $f^{q_{2}}$ and hence that $q_{1} \leq q_{2}$.

Conversely, $q_{1} \leq q_{2}$ means we can divide out by $f^{q_{1}}$, but $f^{q_{2}-q_{1}} I\left(\left(m s-q_{2}\right) Z\right)_{t-2 q_{2}}$ consists only of forms of degree $t-2 q_{1}$ which vanish to order at least $m s-q_{1}$ at each point of $Z$, and hence (since $m s-q_{1} \geq r s-q_{1}$ ) is contained in the complete 
linear system $I\left(\left(r s-q_{1}\right) Z\right)_{t-2 q_{1}}$ of all forms of degree $t-2 q_{1}$ vanishing to order at least $r s-q_{1}$ at each point of $Z$. Multiplying back through by $f^{q_{1}}$ we have

$$
I_{t}^{(m)}=f^{q_{2}} I\left(\left(m s-q_{2}\right) Z\right)_{t-2 q_{2}} \subseteq f^{q_{1}} I\left(\left(r s-q_{1}\right) Z\right)_{t-2 q_{1}}=I^{r}{ }_{t} .
$$

Lemma 3.3. Assume the points $p_{1}, \ldots, p_{n}$ lie on a smooth conic curve $Q^{\prime}$ with $n \geq 5$. Let $I=I(Z)$ for $Z=p_{1}+\cdots+p_{n}$.

(a) If $t \geq 2 m$, then $q\left(I_{t}^{(m)}\right)=\max (0,\lceil(m n-2 t) /(n-4)\rceil)$.

(b) If $t \geq 2 r$ and $n$ is odd, then $q\left(I_{t}^{r}\right)=\max (0,\lceil(r(n+1)-2 t) /(n-3)\rceil)$.

Proof. (a) Just apply Lemma 3.1 with $r=1$ and solve for $s$.

(b) Let $t=t_{1}+\cdots+t_{r}$ with $t_{i} \geq 2$ for all $i$. By (a) with $m=1$ we see that $q\left(I_{t_{i}}\right)$ is 1 for $2 \leq t_{i}<n / 2$ and 0 for $t>n / 2$ ( $t$ cannot equal $n / 2$ since $n$ is odd). Thus $q\left(I_{t_{1}} \cdots I_{t_{r}}\right)=\sum_{i} q\left(I_{t_{i}}\right)$ is the number $s$ of factors $I_{t_{i}}$ for which $t_{i}<n / 2$. Note that there is a product $I_{t_{1}} \cdots I_{t_{r}}$ having exactly $s$ factors $I_{t_{i}}$ with $2 \leq t_{i}<n / 2$ if and only if $s$ satisfies $0 \leq s \leq r$ and $2 s+(r-s)\lceil n / 2\rceil \leq t$, so by Lemma $3.1 q\left(I_{t}^{r}\right)$ is the least $s$ such that $0 \leq s \leq r$ and $2 s+(r-s)\lceil n / 2\rceil \leq t$. Solving $2 s+(r-s)\lceil n / 2\rceil \leq t$ for $s$ using $\lceil n / 2\rceil=(n+1) / 2$ gives $\lceil(r(n+1)-2 t) /(n-3)\rceil \leq s$. We claim that $u=\max (0,\lceil(r(n+1)-2 t) /(n-3)\rceil)$ is the least $s$ such that $0 \leq s \leq r$ and $2 s+(r-s)\lceil n / 2\rceil \leq t$. Note that $2 r \leq t$ implies $\lceil(r(n+1)-2 t) /(n-3)\rceil \leq r$, so $0 \leq u \leq r$. If $u=\lceil(r(n+1)-2 t) /(n-3)\rceil$, then clearly $2 u+(r-u)\lceil n / 2\rceil \leq t$, while if $\lceil(r(n+1)-2 t) /(n-3)\rceil<u=0$, then from $\lceil(r(n+1)-2 t) /(n-3)\rceil<0$ we obtain $2 u+(r-u)\lceil n / 2\rceil=r\lceil n / 2\rceil \leq t$.

Theorem 3.4. Assume the points $p_{1}, \ldots, p_{n}$ lie on a smooth conic curve $Q^{\prime}$. Let $I=I(Z)$, where $Z=p_{1}+\cdots+p_{n}$. Let $m$ and $r$ be positive.

(a) If $n$ is even or $n=1$, then $I^{(m)} \subseteq I^{r}$ if and only if $m \geq r$; in particular, $\rho(I)=1$.

(b) If $n>1$ is odd, then $I^{(m)} \subseteq I^{r}$ if and only if $(n+1) r-1 \leq n m$; in particular, $\rho(I)=(n+1) / n$.

Proof. (a) For any $n>0$, if $I^{(m)} \subseteq I^{r}$, then $I^{m} \subseteq I^{(m)} \subseteq I^{r}$, so $r \alpha(I) \leq m \alpha(I)$. But $Z \neq 0$, so $\alpha(I)>0$; hence $m \geq r$. Conversely, if $n$ is even or $n=1, Z$ is a complete intersection, so $I^{r}=I^{(r)}$ (see the proof of Theorem $32(2)$, p. 110 of $[\mathrm{M}]$ ); hence $I^{(m)} \subseteq I^{(r)}=I^{r}$ if $m \geq r$.

(b) First say $n=3$. Then $\rho(I)=4 / 3$ by $[\mathrm{BH}$, Theorem 4.2.3(a)], $\alpha(I)=$ $\operatorname{reg}(I)$ by [BH, Lemma 2.4.2], and $\alpha\left(I^{(m)}\right)=\lceil 3 m / 2\rceil$ by $[\mathrm{BH}$, Lemma 2.4.1 and Example 4.5]. Thus $I^{(m)} \subseteq I^{r}$ if and only if $2 r=r \alpha(I) \leq \alpha\left(I^{(m)}\right)=\lceil 3 m / 2\rceil$ by Corollary 1.2. But $2 r \leq\lceil 3 m / 2\rceil$ if and only if $4 r \leq 3 m+1$.

Now assume $n \geq 5$. Note that if $(n+1) r-1 \leq n m$, then $m \geq r$ (for if $m<r$, then $(n+1) r-1<n r$; hence $r<1$, contrary to hypothesis), and we saw above that $I^{(m)} \subseteq I^{r}$ implies $m \geq r$. Thus we may assume $m \geq r$. Our result will now follow by Corollary 3.2 (using Lemma 3.3 to compute $q$ ) once we verify that $q\left(I_{t}^{(m)}\right) \geq q\left(I_{t}^{r}\right)$ for all $t \geq 2 m$ if and only if $(n+1) r-1 \leq n m$.

First assume $m$ is even. Since $n$ is odd, $(n+1) r-1 \leq n m$ is equivalent to $(n+1) r \leq n m$. But if $(n+1) r>n m$, then $q\left(I_{t}^{(m)}\right)=0<1 \leq q\left(I_{t}^{r}\right)$ for $t=m n / 2$. Conversely, if $(n+1) r \leq n m$, then $(m n-2 t) /(n-4) \geq(r(n+1)-2 t) /(n-3)$ (and hence $\left.q\left(I_{t}^{(m)}\right) \geq q\left(I_{t}^{r}\right)\right)$ for $2 m \leq t \leq n m / 2$, while for $t>m n / 2$ we have $q\left(I_{t}^{(m)}\right)=q\left(I_{t}^{r}\right)=0($ since both $(m n-2 t) /(n-4)$ and $(r(n+1)-2 t) /(n-3)$ are negative). 
Now assume $m$ is odd. If $(n+1) r-1>n m$, then $q\left(I_{t}^{(m)}\right)=1<2 \leq q\left(I_{t}^{r}\right)$ for $t=((m-1) n+4) / 2$ (note that this implies $t \geq 2 m$, so we can apply Lemma 3.3(a)). Conversely, if $(n+1) r-1 \leq n m$, then $(m n-2 t) /(n-4) \geq(r(n+1)-2 t) /(n-3)$ (and hence $q\left(I_{t}^{(m)}\right) \geq q\left(I_{t}^{r}\right)$ ), for $2 m \leq t \leq((m-1) n+4) / 2$ (this is easy to check since we are comparing two linear functions of $t$ ), while $q\left(I_{t}^{(m)}\right)=1$ and $q\left(I_{t}^{r}\right) \leq 1$ for $((m-1) n+4) / 2<t \leq(m n-1) / 2$, and $q\left(I_{t}^{(m)}\right)=q\left(I_{t}^{r}\right)=0$ for $t \geq(m n+1) / 2$. Thus $I^{(m)} \subseteq I^{r}$ if and only if $(n+1) r-1 \leq n m$. In particular, $m / r \geq(n+1) / n>(n+1) / n-1 /(r n)$ implies $I^{(m)} \subseteq I^{r}$, so $\rho(I) \leq(n+1) / n$. On the other hand, $m / r<(n+1) / n-1 /(r n)$ implies $I^{(m)} \nsubseteq I^{r}$. Since for any ratio $m / r$ less than $(n+1) / n$ we can choose $s \gg 0$ such that $m s /(r s)<(n+1) / n-1 /(r s n)$, we have $I^{(m s)} \nsubseteq I^{r s}$, so $m / r \leq \rho(I)$ and thus $(n+1) / n \leq \rho(I)$; i.e., $\rho(I)=(n+1) / n$.

\section{General points}

In this section we determine $\rho(I)$ and solve the containment problem for each set of $n \leq 9$ general points of $\mathbf{P}^{2}$. Any $n \leq 5$ general points lie on a smooth conic, and hence these cases have been dealt with in the previous section, so now we consider the ideal $I$ of $n$ general points for $6 \leq n \leq 9$. In this section, $X$ will denote the blowup of $\mathbf{P}^{2}$ at these $n$ points. (For each $n$, once we determine exactly when $I^{(m)} \subseteq I^{r}$ occurs, our determination of $\rho(I)$ uses the same argument as used at the end of the proof of Theorem 3.4(b), so we merely state the value of $\rho(I)$ without repeating the justification.)

Proposition 4.1. Let $I$ be the ideal of $n=6$ general points of $\mathbf{P}^{2}$. Then $I^{(m)} \subseteq I^{r}$ if and only if $m \geq \frac{5}{4} r-\frac{5}{12}$, and thus $\rho(I)=\frac{5}{4}$.

Proof. It is easy to see that $\alpha(I)=\operatorname{reg}(I)=3$. Now note that $25 L-10 E$ is nef, where $E=E_{1}+\cdots+E_{6}$, since $25 L-10 E=L+2 \sum_{i}\left(2 L-E+E_{i}\right)$ is the class of a curve with 7 irreducible components (coming from the line and the 6 conics through each subset of 5 of the 6 points) and meets each component non-negatively. Thus $5(5 L-2 E)$ is nef; hence $(\alpha L-m E) \cdot(5 L-2 E) \geq 0$ for $\alpha=\alpha\left(I^{(m)}\right)$. Thus $5 \alpha-12 m \geq 0$ or $\alpha \geq \frac{12 m}{5}$. But $\left\lceil\frac{12 m}{5}\right\rceil L-m E$ is a non-negative integer linear combination of the classes $3 L-E, 5 L-2 E$ and $12 L-5 E$ (just check mod 5), each of which is (by counting constants) effective. Thus $\frac{12 m}{5} \leq \alpha\left(I^{(m)}\right) \leq\left\lceil\frac{12 m}{5}\right\rceil$, so $\alpha\left(I^{(m)}\right)=\left\lceil\frac{12 m}{5}\right\rceil$ and $\gamma(I)=\frac{12}{5}$. Next, note that $m \geq \frac{5}{4} r-\frac{5}{12}$ if and only if $\frac{12 m}{5} \geq$ $3 r-1$ if and only if $\frac{12 m}{5}>3 r-1$ if and only if $\alpha\left(I^{(m)}\right)=\left\lceil\frac{12 m}{5}\right\rceil \geq 3 r=r \operatorname{reg}(I)$. Now apply Corollary 1.2 .

Remark 4.2. The behavior of powers of an ideal $I$ of points is especially simple when, as is the case for $n=6$ general points in $\mathbf{P}^{2}, \alpha(I)=\operatorname{reg}(I)$ : the powers are obtained by truncating homogeneous components of $I^{(r)}$ of low degree. For example, assume that $I \subsetneq k\left[\mathbf{P}^{N}\right]$ is a radical ideal defining a finite non-empty set of points in $\mathbf{P}^{N}$ such that $\alpha(I)=\operatorname{reg}(I)$. Then $I^{r}=I^{(r)} \cap M^{r \alpha}$, where $M=\left(x_{0}, \ldots, x_{N}\right) \subset$ $k\left[\mathbf{P}^{N}\right]$ is the irrelevant ideal and $\alpha=\alpha(I)$ : clearly $I^{r} \subseteq I^{(r)} \cap M^{r \alpha} \subseteq I^{(r)}$ so $I^{r} \subseteq\left(I^{(r)} \cap M^{r \alpha}\right)_{t}=0$ for $t<r \alpha$, while $I_{t}^{r}=I_{t}^{(r)}$ for $t \geq r \alpha=r \operatorname{reg}(\mathrm{I})$ by $\mathrm{BH}$, Lemma 2.3.3(c)].

Proposition 4.3. Let $I$ be the ideal of $n=7$ general points of $\mathbf{P}^{2}$. Then $I^{(m)} \subseteq I^{r}$ if and only if either $m \geq \frac{8}{7}$ r or $r=m=1$, and thus $\rho(I)=\frac{8}{7}$. 
Proof. Let $E=E_{1}+\cdots+E_{7}$ and let $C$ be a general (hence smooth and irreducible) curve $C \in\left|-K_{X}\right|$; thus $[C]=[3 L-E]$.

First, note for each $i$ that $C-E_{i}$ is linearly equivalent to an effective divisor $C_{i}$ (by counting constants) which is reduced and irreducible (otherwise $C-E_{i}$ would be a sum of two or more effective divisors, at least one of which would come from either a line through 3 or more points or a conic through 6 or more points, but neither can happen since the points are general). Thus $3(8 L-3 E)=C+\sum_{i} C_{i}$ is nef since it meets each component non-negatively; hence $(\alpha L-m E) \cdot(8 L-3 E) \geq 0$ for $\alpha=\alpha\left(I^{(m)}\right)$. Thus $8 \alpha-21 m \geq 0$, so $\alpha \geq\left\lceil\frac{21 m}{8}\right\rceil$. But $\left\lceil\frac{21 m}{8}\right\rceil L-m E$ is a nonnegative integer linear combination of the classes $3 L-E, 8 L-3 E$ and $21 L-8 E$ (just check mod 8), each of which is effective. Thus $\alpha \leq\left\lceil\frac{21 m}{8}\right\rceil$, so $\alpha=\left\lceil\frac{21 m}{8}\right\rceil$.

We claim that $I_{t}^{r}=I_{t}^{(r)}$ for all $t \geq 3 r+1$. To justify this, it is enough to show that $\left(I_{3}\right)^{r-1}\left(I_{t-3 r+3}\right)=I_{t}^{(r)}$, i.e., that $H^{0}\left(X,-K_{X}\right)^{\otimes(r-1)} \otimes H^{0}\left(X, i L-K_{X}\right) \rightarrow$ $H^{0}\left(X, i L-r K_{X}\right)$ is onto for each $i>0$ and each $r \geq 1$. To prove this, it is enough in fact to show that $H^{0}\left(X,-K_{X}\right) \otimes H^{0}\left(X, i L-m K_{X}\right) \rightarrow H^{0}\left(X, i L-(m+1) K_{X}\right)$ is onto for each $i>0$ and each $m \geq 0$. Now consider the following diagram, where $V_{F}=H^{0}(X, F)$ and the vertical maps are the canonical multiplication maps:

$$
\begin{array}{ccccccccc}
0 & \rightarrow & H^{0}(X, G-C) \otimes V_{F} & \rightarrow & H^{0}(X, G) \otimes V_{F} & \rightarrow & H^{0}\left(C,\left.G\right|_{C}\right) \otimes V_{F} & \rightarrow & 0 \\
& & \downarrow^{\mu_{1}} & & & \downarrow \mu_{2} & & & \downarrow \\
0 & \rightarrow & H^{0}(X, F+G-C) & \rightarrow & H^{0}(X, F+G) & \rightarrow & H^{0}\left(C,\left.(F+G)\right|_{C}\right) & \rightarrow & 0
\end{array}
$$

Consider the case that $F=-K_{X}$ and $G=i L-m K_{X}$. To see that the rows of the diagram are exact, note that since $C$ is a prime divisor of non-negative self-intersection, it is nef; hence so are $-m K_{X}$ and $i L-m K_{X}$ for any $i>0$ and $m \geq 0$, and hence $h^{1}\left(X,-m K_{X}\right)=0$ and $h^{1}\left(X, i L-m K_{X}\right)=0$ by [H5]. Note when $m=1$ that $\mu_{1}$ (i.e., $H^{0}(X, i L) \otimes H^{0}\left(X,-K_{X}\right) \rightarrow H^{0}\left(X, i L-K_{X}\right)$ ) is onto by [H3]. If we show that $\mu_{3}$ is onto for each $m \geq 1$, then the snake lemma applied to the diagram and induction on $m$ show that $\mu_{2}$ is onto for all $m \geq 1$. (We can at least see that $\mu_{3}$ is onto for $m=0$ : apply the snake lemma to the diagram above with $F=i L$ and $G=-K_{X}$, using the fact that in this case $\mu_{2}$ is $H^{0}\left(X,-K_{X}\right) \otimes H^{0}(X, i L) \rightarrow H^{0}\left(X, i L-K_{X}\right)$, which we just noted is onto. $)$

To show that $\mu_{3}$ is onto, let $Z \in|C|_{C} \mid$ be a divisor on $C$ consisting of two distinct points and consider the diagram

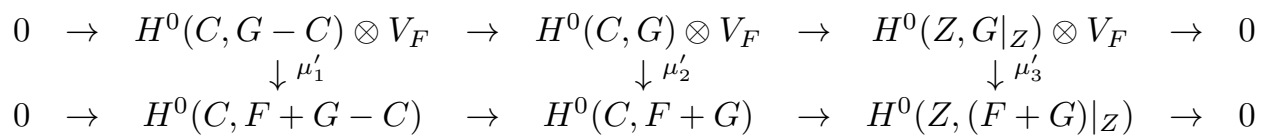

where now $F=-\left.K_{X}\right|_{C}, V_{F}=H^{0}(C, F)$ and $G=\left.\left(i L-m K_{X}\right)\right|_{C}$. The map $\mu_{1}^{\prime}$ is onto for $m=1$ by the parenthetical remark at the end of the preceding paragraph. Thus the snake lemma applied to the new diagram, using induction on $m$, implies $\mu_{2}^{\prime}$ is onto for all $m \geq 1$ if we show $\mu_{3}^{\prime}$ is onto. Since $0 \rightarrow \mathcal{O}_{X} \rightarrow \mathcal{O}_{X}\left(-K_{X}\right) \rightarrow$ $\mathcal{O}_{C}\left(-K_{X}\right) \rightarrow 0$ is exact on global sections the map $H^{0}(X, F) \rightarrow H^{0}\left(C,\left.F\right|_{C}\right)$ is surjective; hence $\mu_{2}^{\prime}$ and $\mu_{3}$ have the same image. Thus surjectivity of $\mu_{2}^{\prime}$ implies that of $\mu_{3}$, which is what we want to show. To see that $\mu_{3}^{\prime}$ is onto, note that $H^{0}\left(Z,\left.(F+G)\right|_{Z}\right)$ is just the vector space of $k$-valued functions on the two points of $Z$, as is $H^{0}\left(Z,\left.G\right|_{Z}\right)$, and $\mu_{3}^{\prime}$ just multiplies a function in $H^{0}\left(Z,\left.G\right|_{Z}\right)$ by the values 
of an element of $V_{F}$ at the two points. But $\left|-K_{X}\right|_{C} \mid$ is base point free (since a complete linear system of degree 2 on an elliptic curve $C$ is base point free); i.e., $V_{F}=H^{0}(C, F)$ is base point free and hence there is an element of $V_{F}$ which is nonzero at both points, so $\mu_{3}^{\prime}$ is onto, which finishes our justification that $I_{t}^{r}=I_{t}^{(r)}$ for all $t \geq 3 r+1$.

We now proceed to find all $m>0$ for each $r>0$ such that $I^{(m)} \subseteq I^{r}$. If $\left\lceil\frac{21 m}{8}\right\rceil<3 r$, then $\alpha\left(I^{(m)}\right)<\alpha\left(I^{r}\right)$, so $I^{(m)} \nsubseteq I^{r}$. If $\left\lceil\frac{21 m}{8}\right\rceil \geq 3 r$, then $m \geq r$ (if not, then $r>m$, but $\left\lceil\frac{21 m}{8}\right\rceil \geq 3 r$ implies $\frac{21 m}{8}>3 r-1$ and so $\frac{8}{3}>8 r-7 m$ which with $r>m$ gives $\frac{8}{3}>8 r-7 m>r$, so $r=1$ and $m=0$, contrary to hypothesis), so $I^{(m)} \subseteq I^{(r)}$. Now suppose $\left\lceil\frac{21 m}{8}\right\rceil>3 r$; then $I^{(m)} \subseteq I^{r}$, since $0=I_{t}^{(m)} \subseteq I^{r}$ for $t<\alpha\left(I^{(m)}\right)=\left\lceil\frac{21 m}{8}\right\rceil$, and $t \geq \alpha\left(I^{(m)}\right)=\left\lceil\frac{21 m}{8}\right\rceil$ implies $t \geq 3 r+1$, so $I_{t}^{(m)} \subseteq I_{t}^{(r)}=I^{r}$.

So suppose $\alpha=\left\lceil\frac{21 m}{8}\right\rceil=3 r$. By the same arguments, $I_{t}^{(m)} \subseteq I_{t}^{r}$ if either $t<3 r$ or $t>3 r$, so $I^{(m)} \subseteq I^{r}$ if and only if $I_{3 r}^{(m)} \subseteq I_{3 r}^{r}$. By checking mod 8, we can see that $\left\lceil\frac{21 m}{8}\right\rceil=3 r$ implies $m$ is congruent to 0,1 or 2 modulo 8 . If $m \equiv 0 \bmod 8$, then $m=8 i$ for some $i$, so $r=7 i$ and $\alpha=21 i$. But $|\alpha L-m E|=\left|i \sum_{j} C_{j}\right|=i \sum_{j} C_{j}$ is fixed, since the curves $C_{j}$ are disjoint prime divisors of negative self-intersection. Thus $I_{\alpha}^{(m)}$ is 1-dimensional, spanned by the product of the $r=7 i$ cubic forms corresponding to the summands of $i \sum_{j} C_{j}$, with each cubic form vanishing at each of the $n=7$ points and hence being in $I_{3}$. Thus $I_{\alpha}^{(m)} \subseteq I_{\alpha}^{r}$.

Say $m \equiv 1 \bmod 8$, so $m=8 i+1$ and $r=7 i+1$ for some $i$. If $m=1$, then clearly $I=I^{(m)} \subseteq I^{r}$ if and only if $r=1$, so say $m>1$ and hence $i \geq 1$. Then $I_{3 r}^{(m)}=H^{0}\left(X, 3(8 L-3 E)+(i-1) \sum_{j} C_{j}\right)$; note that $\mid 3(8 L-3 E)+(i-$ 1) $\sum_{j} C_{j}|=| 3(8 L-3 E) \mid+(i-1) \sum_{j} C_{j}$ since the $C_{j}$ are disjoint and of negative self-intersection with $(8 L-3 E) \cdot C_{j}=0$, so $(i-1) \sum_{j} C_{j}$ is fixed in the linear system. Let $C$ be a smooth section of $\left|-K_{X}\right|$. By Serre duality, $h^{1}\left(X, \mathcal{O}_{X}(3(8 L-\right.$ $3 E)-C))=h^{1}\left(X, \mathcal{O}_{X}(-(3(8 L-3 E)))\right)$ and since $3(8 L-3 E)$ is nef and big, $h^{1}\left(X, \mathcal{O}_{X}(-(3(8 L-3 E)))\right)=0$ by Ramanujam vanishing (for the characteristic $p$ version, see Theorem 1.6 [T] or Theorem 2.8 of [H1]). Thus $0 \rightarrow \mathcal{O}_{X}(3(8 L-3 E)-$ $C) \rightarrow \mathcal{O}_{X}(3(8 L-3 E)) \rightarrow \mathcal{O}_{C}(3(8 L-3 E)) \rightarrow 0$ is exact on global sections, and $\mathcal{O}_{C}(3(8 L-3 E))$ is very ample (since it has degree 9 , and any divisor of degree at least 3 on an elliptic curve is very ample). In particular, the trace of $|3(8 L-3 E)|$ on $C$ is not composed with a pencil. Since the trace of $I_{3 r}^{r}=\left(I_{3}\right)^{r}$ on $C$ is composed with the pencil given by the trace of $\left|-K_{X}\right|$ on $C$, we see that $I_{3 r}^{(m)} \nsubseteq I_{3 r}^{r}$, and hence $I^{(m)} \nsubseteq I^{r}$.

Finally, say $m \equiv 2 \bmod 8$ (i.e., $m=8 i+2$ and $r=7 i+2$ for some $i$ ). Then $I_{3 r}^{(m)}=$ $H^{0}\left(X, 6(8 L-3 E)+(i-2) \sum_{i} C_{i}\right)$ for $i \geq 2$, while $I_{3 r}^{(m)}=H^{0}(X, 3(8 L-3 E)+C)$ for $i=1$ and $I_{3 r}^{(m)}=H^{0}(X, 2 C)$ for $i=0$. Arguing as before, the trace of $I_{3 r}^{(m)}$ on $C$ is not composed with a pencil, but the trace of $I_{3 r}^{r}$ on $C$ is composed with the same pencil as before. Thus $I_{3 r}^{(m)} \nsubseteq I_{3 r}^{r}$, so $I^{(m)} \nsubseteq I^{r}$.

Reviewing, we have $I^{(m)} \subseteq I^{r}$ if and only if $m=r=1$, or $\left\lceil\frac{21 m}{8}\right\rceil>3 r$ (which is equivalent to $\frac{21 m}{8}>3 r$, i.e., to $m / r>8 / 7$ ), or $m=8 i$ and $r=7 i$ for $i \geq 1$ (which is equivalent to $m / r=8 / 7$ ). Thus $I^{(m)} \subseteq I^{r}$ if and only if $m=r=1$ or $m / r \geq 8 / 7$. 
The following result is interesting in that early evidence suggested that $\rho(I) \leq \sqrt{2}$ for $n$ generic points of $\mathbf{P}^{2}$ (see, for example, $\overline{\mathrm{BH}}$, Corollary 1.3.1, Proposition 4.4]). For $n=8$, however, we now see that $\rho(I)>\sqrt{2}$ (but only by a bit).

Proposition 4.4. Let $I$ be the ideal of $n=8$ general points of $\mathbf{P}^{2}$. Then $I^{(m)} \subseteq I^{r}$ if and only if either $m=r=1$ or $m \geq \frac{17}{12} r-\frac{1}{3}$, and thus $\rho(I)=\frac{17}{12}$.

Proof. Let $C^{\prime}$ be a general cubic through the 8 general points $p_{i}$. Thus we may assume that $C^{\prime}$ is smooth and the class of its proper transform $C$ is $-K_{X}$. Since $C^{2}=1 \geq 0$, we see that $-K_{X}$ is nef.

Now $\alpha=\alpha\left(I^{(m)}\right)=\left\lceil\frac{48 m}{17}\right\rceil$ : let $F=\left(\left\lceil\frac{48 m}{17}\right\rceil\right) L-m E$, where $E=E_{1}+\cdots+$ $E_{8}$. Then $F$ is a non-negative integer linear combination of $-K_{X}, 17 L-6 E$ and $48 L-17 E$, and these all (by counting constants) are effective. Thus $\alpha \leq\left\lceil\frac{48 m}{17}\right\rceil$. But $17 L-6 E$ is nef (since it reduces by quadratic Cremona transformations to $L$ [H6]), so $(\alpha L-m E) \cdot(17 L-6 E) \geq 0$; hence $\alpha \geq\left\lceil\frac{48 m}{17}\right\rceil$.

Next, $\alpha \geq 4 r$ if and only if $\frac{48 m}{17}>4 r-1$ if and only if $48 m>68 r-17$ if and only if $48 m \geq 68 r-16$ if and only if $m \geq \frac{17}{12} r-\frac{1}{3}$. Note also that $\operatorname{reg}(I)=4$. Thus, if $m \geq \frac{17}{12} r-\frac{1}{3}$, then $\alpha\left(I^{(m)}\right) \geq 4 r=r \operatorname{reg}(I)$, so (by [BH, Lemma 2.3.4]) $I^{(m)} \subseteq I^{r}$. Also, if $m=r=1$, then $I^{(m)}=I=I^{r}$.

Conversely, assume $m<\frac{17}{12} r-\frac{1}{3}$; hence $\alpha\left(I^{(m)}\right)<4 r$. If $m=1$ and $r>1$, then $\alpha\left(I^{(m)}\right)=3<3 r=\alpha\left(I^{r}\right)$, so $I^{(m)} \nsubseteq I^{r}$. So assume $m>1$ and $t=\alpha\left(I^{(m)}\right)$; we will show that $I_{t}^{r}$ has a base point which $I_{t}^{(m)}$ doesn't have. It thus follows that $I^{(m)} \nsubseteq I^{r}$.

If $t=\alpha\left(I^{(m)}\right)<r \alpha(I)=3 r$, this is clear, since $I_{t}^{(m)} \neq 0$ but $I_{t}^{r}=0$. So assume $r \alpha(I) \leq t=\alpha\left(I^{(m)}\right)<4 r$; i.e., assume $3 r-1<48 m / 17 \leq 4 r-1$. Note that $I^{r}{ }_{t}=\sum_{t_{1} \leq \cdots \leq t_{r}} \prod_{i} I_{t_{i}}$, where the sum is over all sequences $t_{i}$ with $3 \leq t_{1} \leq \cdots \leq t_{r}$ such that $\sum_{i} t_{i}=t$ (we ignore sequences with $t_{1}<3$ since $I_{j}=0$ for $j<3$ ). Since $t<4 r$, we see that $t_{1}=3$ for every sequence; hence $\prod_{i} I_{t_{i}}$ and thus $I_{t}^{r}$ has the same base points as does $I_{3}$, these being the nine base points $p_{1}, \ldots, p_{9}$ of the pencil of cubics $I_{3}$ through our $n=8$ general points $p_{1}, \ldots, p_{8}$. So now it suffices to show that $p_{9}$ is not a base point of $I_{t}^{(m)}$.

First we check that the points $p_{1}, \ldots, p_{9}$ are distinct. Note that $p_{9}$ can be identified with the base point of $|C|$ (i.e., under the morphism $\pi: X \rightarrow \mathbf{P}^{2}$ blowing up the 8 points, if $p$ is the base point of $|C|$, then $\left.\pi(p)=p_{9}\right)$. Let $q_{i}$ be the point where $E_{i}$ meets $C$; then $\pi\left(q_{i}\right)=p_{i}$. Thus to show that $p_{i} \neq p_{9}$ for $i<9$, it is enough to show that $q_{i} \neq p$, i.e., that the restriction of $C-E_{i}$ to $C$ is not trivial, which is the same as showing that $h^{0}\left(C, \mathcal{O}_{C}\left(C-E_{i}\right)\right)=0$, since a line bundle of degree 0 on an elliptic curve has positive $h^{0}$ if and only if the line bundle is trivial (in which case $\left.h^{0}=1\right)$. So suppose that $h^{0}\left(C, \mathcal{O}_{C}\left(C-E_{i}\right)\right)=$ 1. Note $h^{0}\left(X, \mathcal{O}_{X}\left(-E_{i}\right)\right)=0$ (since $L-E_{i}$ is nef and $\left(L-E_{i}\right) \cdot\left(-E_{i}\right)<0$ ), $h^{2}\left(X, \mathcal{O}_{X}\left(-E_{i}\right)\right)=h^{0}\left(X, \mathcal{O}_{X}\left(K_{X}+E_{i}\right)\right)=0$ (by duality and the facts that $L$. $\left(K_{X}+E_{i}\right)<0$ and that $L$ is nef) and $h^{1}\left(X, \mathcal{O}_{X}\left(-E_{i}\right)\right)=0$ (by Riemann-Roch since $h^{0}\left(X, \mathcal{O}_{X}\left(-E_{i}\right)\right)-h^{1}\left(X, \mathcal{O}_{X}\left(-E_{i}\right)\right)+h^{2}\left(X, \mathcal{O}_{X}\left(-E_{i}\right)\right)=\left(\left(-E_{i}\right)^{2}-K_{X} \cdot\left(-E_{i}\right)\right) / 2+$ $1=0)$. Taking cohomology of the exact sequence $0 \rightarrow \mathcal{O}_{X}\left(-E_{i}\right) \rightarrow \mathcal{O}_{X}\left(C-E_{i}\right) \rightarrow$ $\mathcal{O}_{C}\left(C-E_{i}\right) \rightarrow 0$, we now see that $h^{0}\left(X, \mathcal{O}_{X}\left(C-E_{i}\right)\right)=h^{0}\left(C, \mathcal{O}_{C}\left(C-E_{i}\right)\right)=1$. For $i=1$ (just to be specific, the argument for $1<i \leq 8$ being the same) we have $C-E_{1}=3 L-2 E_{1}-E_{2}-\cdots-E_{8}$. But $h^{0}\left(X, \mathcal{O}_{X}\left(3 L-2 E_{1}\right)\right)=\operatorname{dim} I\left(2 p_{1}\right)_{3}=7$, and for a general point $p_{j+1}$ with $j \geq 1$, the dimension of $\mid 3 L-2 E_{1}-E_{2}-\cdots-$ $E_{j+1} \mid$ is one less than the dimension of $\left|3 L-2 E_{1}-E_{2}-\cdots-E_{j}\right|$ as long as 
$\left|3 L-2 E_{1}-E_{2}-\cdots-E_{j}\right|$ is not empty (since assigning a general base point to any non-empty linear system imposes exactly one condition). Thus $h^{0}\left(X, \mathcal{O}_{X}\left(C-E_{i}\right)\right)$ is 0 for general points, contradicting $h^{0}\left(C, \mathcal{O}_{C}\left(C-E_{i}\right)\right)=1$.

It is well known that the divisor class $\left[2 C-E_{i}\right]=\left[6 L-2\left(E_{1}+\cdots+e_{8}\right)-E_{i}\right]$ is the class of a smooth irreducible curve $D_{i}$ when the points $p_{1}, \ldots, p_{8}$ are general; indeed, in this situation, $\left[2 C-E_{i}\right]$ reduces by quadratic Cremona transformations to $\left[E_{1}\right]$ (see [H6], for example). Hence $D_{i}$ is a smooth rational curve of self-intersection -1 which meets $C$ at a single point $q_{i}^{\prime}$, since $C \cdot D_{i}=1$. As before, $p \neq q_{i}^{\prime}$. (If $p=q_{i}^{\prime}$, then $\mathcal{O}_{C}\left(D_{i}-C\right)$, which is equal to $\mathcal{O}_{C}\left(C-E_{i}\right)$, would be trivial, and we have seen that it is not.)

Under the identification of $I_{t}^{(m)}$ with $H^{0}(X, F)$ for $F=t L-m E$, if $|F|$ has a base point $p^{\prime}$, then $p^{\prime \prime}=\pi\left(p^{\prime}\right)$ is a base point of $I_{t}^{(m)}$. Not every base point of $I_{t}^{(m)}$ comes from a base point of $|F|$, since $p_{1}, \ldots, p_{8}$ are always base points of $I_{t}^{(m)}$ even though $|F|$ can sometimes be base point free (after all, one of the motivations historically for blowing up points was to remove base points). However, if $I_{t}^{(m)}$ has a base point $p^{\prime \prime}$ away from the points $p_{1}, \ldots, p_{8}$ blown up by $\pi$, then $|F|$ has a base point $p^{\prime}$ with $\pi\left(p^{\prime}\right)=p^{\prime \prime}$. Thus, since the points $p_{1}, \ldots, p_{9}$ are distinct, $p_{9}$ is a base point of $I_{t}^{(m)}$ if and only if $p$ is a base point of $|F|$. But, as we saw above, $F=a C+b(17 L-6 E)+c(48 L-17 E)$ for some non-negative integers $a, b$ and $c$. Since we are assuming that $m>1$, either $a>1$, or $b$ or $c$ is positive. If $b>0$, then $a C+b(17 L-6 E)$ is nef with $(a C+b(17 L-6 E)) \cdot C>1$, so $|a C+b(17 L-6 E)|$ is base point free by [H1. Also $48 L-17 E=D_{1}+\cdots+D_{8}$, so $p$ is not a base point of $|48 L-17 E|$ and hence not of $|F|$ when $b>0$ (since then we can write $F$ as a sum of effective divisors, none of which pass through $p$ ). So suppose $b=0$. If $a>1$, then $|a C|$ is nef with $a C \cdot C>1$, hence base point free by [H1, so the same argument again shows $p$ is not a base point of $|F|$. If $a=1$ but $c>0$, we reduce to the case that $b>0$, since $F=(C+(48 L-17 E))+(c-1)(48 L-17 E)=$ $3(17 L-6 E)+(c-1)(48 L-17 E)$.

In stating the next result, one needs to be careful. The issue is that the condition of generality on the points which guarantees that the result holds depends on $m$. The reason is that for 9 points, $h^{0}\left(X,-m K_{X}\right)=1$ holds for general points, but the open condition for which this holds becomes smaller as $m$ increases.

Proposition 4.5. Fix positive integers $m$ and $r$. Then $I^{(m)} \subseteq I^{r}$, where $I$ is the ideal of $n=9$ general points of $\mathbf{P}^{2}$ if and only if $m \geq \frac{4}{3} r-\frac{1}{3}$. For $n=9$ generic points we thus have $\rho(I)=\frac{4}{3}$.

Proof. Let $F=\alpha L-m E$ for $E=E_{1}+\cdots+E_{9}$ and $\alpha=\alpha\left(I^{(m)}\right)$. Let $C^{\prime}$ be a general cubic curve through $p_{1}, \ldots, p_{9}$ and let $C$ be its proper transform. Then $C$ is smooth and irreducible with $C^{2}=0$, hence nef. Thus $F \cdot C \geq 0$ implies $\alpha \geq 3 m$, but $3 m L-m E=m C$, so $\alpha \leq 3 m$. Thus $\alpha\left(I^{(m)}\right)=3 m$ but, for $t \geq 3 m, I_{t}^{(m)}$ is fixed component free (by [H6]) if and only if $t \geq 3 m+1$, whereas for $t \geq \alpha\left(I^{r}\right)=3 r$, $I^{r}{ }_{t}$ is fixed component free if and only if there is a product $I_{t_{1}} \cdots I_{t_{r}}$ for a sequence $t_{1} \leq \cdots \leq t_{r}$ with $\sum_{i} t_{i}=t$ with $t_{1}>3$, i.e., if and only if $t \geq 4 m$. Since $L-K_{X}$ is normally generated $\mathrm{H} 1$ and $\left|i L-K_{X}\right|$ is fixed component free [H2, Proposition 3.2.1.1(b)] and $H^{0}\left(X, L-K_{X}\right) \otimes H^{0}(X, i L) \rightarrow H^{0}\left(X,(i+1) L-K_{X}\right)$ is onto [H2, Theorem 3.2.1.2(b)] for $i>0$, we have $I_{t_{1}} \cdots I_{t_{r}}=I_{t}^{(r)}$ for all sequences $4 \leq t_{1} \leq \cdots \leq t_{r}$ with $\sum_{i} t_{i}=t \geq 4 r$ and hence $I_{t}^{r}=I_{t}^{(r)}$. Thus $\alpha\left(I^{(m)}\right) \leq 4 r-2$ 
implies $I^{(m)} \nsubseteq I^{r}$ (since there is a $t$ with $\alpha\left(I^{(m)}\right)=3 m<t<4 r$, so $I_{t}^{(m)}$ has no fixed components but $I^{r}$ does; hence $\left.I_{t}^{(m)} \nsubseteq I_{t}^{r}\right)$. If $\alpha\left(I^{(m)}\right) \geq 4 r$, then $I^{(m)} \subseteq I^{(r)}$ by $\left[\mathrm{BH}\right.$, Lemma 2.3.4] since $\operatorname{reg}(I)=4$. Finally, if $3 m=\alpha\left(I^{(m)}\right)=4 r-1$, then $I^{(m)} \subseteq I^{r}$, since $I_{t}^{(m)} \subseteq I^{r}{ }_{t}$ for all $t \geq 0$. To see this, note first that $3 m=4 r-1$ implies $m \geq r$, so $I^{(m)} \subseteq I^{(r)}$. Also note that $I_{t}^{(r)}=I_{t}^{r}$ for $t \geq 4 r$ by $[\mathrm{BH}$, Lemma 2.3.3(c)] and that $I_{3 m}^{(m)}=I_{3 m}^{m}$, since $h^{0}\left(X,-m K_{X}\right)=1$, so $\left|-m K_{X}\right|=$ $m C$. Now we see that $I_{t}^{(m)} \subseteq I_{t}^{r}$ for $t<\alpha\left(I^{(m)}\right)$ (since $I_{t}^{(m)}=0$ ), for $t=\alpha\left(I^{(m)}\right.$ ) (since $I_{3 m}^{(m)}=I_{3 m}^{m}$ and $m \geq r$, so $I_{3 m}^{m} \subseteq I_{4 r-1}^{r}$ ), and for $t>\alpha\left(I^{(m)}\right)$ (since $\left.I_{t}^{(m)} \subseteq I_{t}^{(r)}=I^{r}\right)$. Thus $I^{(m)} \subseteq I^{r}$ if and only if $3 m=\alpha\left(I^{(m)}\right) \geq 4 r-1$ if and only if $m \geq \frac{4}{3} r-\frac{1}{3}$.

\section{EXAMPLES AND QUESTIONS}

It is an interesting problem to determine for which ideals we have $I^{(r)}=I^{r}$ for all $r \geq 1$. It follows by Macaulay's Unmixedness Theorem that $I^{(r)}=I^{r}$ holds for complete intersections (see the proof of Theorem 32 (2), p. 110 of $[\mathrm{M}]$ ). More recently, characterizations of ideals for which symbolic and ordinary powers coincide have been given by [Ho] (for prime ideals) and by [LS] (for radical ideals). Such ideals have been studied also in [MNV], $[\mathrm{Mo}$ and $[\mathrm{HH}]$. In the following example we give constructions of ideals for which $I^{(r)}=I^{r}$ for all $r \geq 1$; these seem to be new.

Example 5.1. Consider a fat point subscheme coming from the class of a smooth rational curve of self-intersection 0 on the blowup $X$ of $\mathbf{P}^{2}$ at general points $p_{1}, \ldots, p_{n} \in \mathbf{P}^{2}$. There are many additional examples which can be obtained from the one below by using the action of the Cremona group. For specificity, let $d$ be a positive integer bigger than 2 , let $n=2 d$, and let $p_{1}, \ldots, p_{n} \in \mathbf{P}^{2}$ be general points. Let $Z=(d-1) p_{1}+p_{2}+\cdots+p_{n}$. Then $F=d L-(d-1) E_{1}-E_{2}-\cdots-E_{n}$ is (linearly equivalent to) an effective divisor by Riemann-Roch; the general member of $|F|$ is a smooth rational curve, the proper transform $D$ in fact of an irreducible degree $d$ curve $C^{\prime}$ with a singularity of multiplicity $d-1$ (the curve $C^{\prime}$ can be obtained by applying quadratic Cremona transformations to a line in $\mathbf{P}^{2}$ ). It follows that $\alpha(I(Z)) \leq d$ but $F$ is nef with $F^{2}=0$, so $\alpha(I(Z)$ ) cannot be less than $d$ (since $\alpha(I(Z))<d$ implies that $F-L$ is effective, but $F \cdot(F-L)=-d<0$, which is impossible since $F$ is nef).

Consider $0 \rightarrow \mathcal{O}_{X}((m-1) F) \rightarrow \mathcal{O}_{X}(m F) \rightarrow \mathcal{O}_{D}(m F) \rightarrow 0$. The restriction $\left.(m F)\right|_{D}$ is trivial, since $D$ is smooth and rational and $F^{2}=0$. By induction on $m$, taking the cohomology of $0 \rightarrow \mathcal{O}_{X}((m-1) F) \rightarrow \mathcal{O}_{X}(m F) \rightarrow \mathcal{O}_{D} \rightarrow 0$ and using the fact that $h^{1}\left(X, \mathcal{O}_{X}\right)=0$, we see that $h^{1}\left(X, \mathcal{O}_{X}(m F)\right)=0$ for all $m \geq 0$. It now follows by induction that $h^{0}\left(X, \mathcal{O}_{X}(m F)\right)=m+1$. A similar argument applied to $0 \rightarrow \mathcal{O}_{X}((m-1) F+L) \rightarrow \mathcal{O}_{X}(m F+L) \rightarrow \mathcal{O}_{D}(L) \rightarrow 0$ gives $h^{1}\left(X, \mathcal{O}_{X}(m F+L)\right)=0$ and $h^{0}\left(X, \mathcal{O}_{X}(m F+L)\right)=m(d+1)+3$.

Consider the following diagram, where $V_{L}=H^{0}(X, L)$ and the vertical maps are the canonical multiplication maps:

$$
\begin{gathered}
0 \rightarrow H^{0}(X,(m-1) F) \otimes V_{L} \rightarrow H^{0}(X, m F) \otimes V_{L} \rightarrow H^{0}\left(D,\left.(m F)\right|_{D}\right) \otimes V_{L} \rightarrow 0 \\
\downarrow^{\mu_{1}} \quad \downarrow^{\mu_{2}} \\
\downarrow^{\mu_{3}} \\
0 \rightarrow H^{0}(X,(m-1) F+L) \rightarrow H^{0}(X, m F+L) \rightarrow H^{0}\left(D,\left.(m F+L)\right|_{D}\right) \rightarrow 0
\end{gathered}
$$


By induction on $m$ using the snake lemma and the fact that $d>2$, the maps $\mu_{1}, \mu_{2}$ and $\mu_{3}$ are injective; hence we have exact sequences $0 \rightarrow \operatorname{cok}\left(\mu_{1}\right) \rightarrow \operatorname{cok}\left(\mu_{2}\right)$ $\rightarrow \operatorname{cok}\left(\mu_{3}\right) \rightarrow 0$ and $0 \rightarrow \operatorname{Im}\left(\mu_{1}\right) \rightarrow \operatorname{Im}\left(\mu_{2}\right) \rightarrow \operatorname{Im}\left(\mu_{3}\right) \rightarrow 0$. Since $h^{0}\left(X, \mathcal{O}_{X}(F)\right)=$ 2 , there is a section $C \in|F|$ disjoint from $D$. Taking $m=1$, let $C_{1}, \ldots, C_{d-2}$ be sections of $|F+L|$ which span a subspace complementary to the image of $\mu_{2}$. Let $L_{1}, L_{2}$ and $L_{3}$ be a basis for $V_{L}$. By induction we find for every $m$ that the image of $\mu_{2}$ is spanned by $i C+j D+L_{k}$ for $0 \leq i, j \leq m, i+j=m$ and $1 \leq k \leq 3$, and $H^{0}(X, m F+L)$ is spanned by these together with $i^{\prime} C+j^{\prime} D+C_{l}, 0 \leq i^{\prime}, j^{\prime} \leq m-1$, $i^{\prime}+j^{\prime}=m-1,1 \leq l \leq d-2$. (For the induction, note that the basis for $H^{0}(X,(m+1) F+L)$ comes partly from the basis for $H^{0}(X, m F+L)$, by adding $D$ to the basis elements of $H^{0}(X, m F+L)$, and partly from $H^{0}\left(C,\left.((m+1) F+L)\right|_{D}\right) \cong$ $H^{0}\left(C,\left.L\right|_{D}\right)$, where the isomorphism takes the restriction of $m C+C_{i}$ to that of $C_{i}$.)

Expressing the foregoing in terms of ideals, we have that the regularity of $I(\mathrm{mZ})$ is at most $m \alpha+1$ and thus that $I(m Z)$ is generated in degrees $m \alpha$ and $m \alpha+1$ and, as long as $d>2$, both degrees are needed (where we denote $\alpha(I(Z)$ ) simply by $\alpha$ ). Elements $A$ and $B$, corresponding to $C$ and $D$ above, span $I(Z)_{\alpha}$, and $C_{1}^{\prime}, \ldots, C_{d-2}^{\prime}$, corresponding to the $C_{i}$ above, span a subspace of $I(Z)_{\alpha+1}$ complementary to the image of $I(Z)_{\alpha} \otimes R_{1} \rightarrow I(Z)_{\alpha+1}$, where $R=k\left[\mathbf{P}^{2}\right]$, so $R_{1}$ denotes the vector space span of the linear forms. Then $A^{i} B^{m-i}$ for $0 \leq i \leq m \operatorname{span} I(m Z)_{\alpha}$, and $A^{i} B^{m-i-1} C_{j}^{\prime}$ for $0 \leq i \leq m-1$ and $1 \leq j \leq d-2$ span a subspace of $I(m Z)_{m \alpha+1}$ complementary to the image of $I(Z)_{m \alpha}^{m} \otimes R_{1} \rightarrow I(Z)_{m \alpha+1}$. Thus the elements $A^{i} B^{m-i}$ and $A^{i} B^{m-i-1} C_{j}^{\prime}$ generate the ideal $I(m Z)$, but all of these elements are in $I(Z)^{m}$, and hence $I(Z)^{m} \subseteq I(m Z) \subseteq I(Z)^{m}$; i.e., $I(Z)^{m}=I(m Z)=I(Z)^{(m)}$, and clearly there is no $m \geq 1$ such that $I(m Z)$ is a power of any ideal which is prime (since the support of $m Z$ consists of a finite set with more than one point), radical (since the points do not all have the same multiplicity), or a complete intersection (since if $I(m Z)=J^{r}$ for some complete intersection $J$, then $I(m s Z)=J^{r s}$ for all $s \geq 1$ and hence $J^{s r}$ is generated either in one degree or in a range of degrees that increases with $s$, but $I(m s Z)$ is generated in two degrees for all $s$ ).

If $I$ is homogeneous with $0 \neq I \subsetneq k\left[\mathbf{P}^{N}\right]$ and $I^{(r)}=I^{r}$ for all $r \geq 1$, then $\rho(I)=1$. However, we do not know any examples with $\rho(I)=1$ but for which $I^{(r)}=I^{r}$ fails for some $r$. This raises the following question:

Question 5.2. Let $0 \neq I \subsetneq k\left[\mathbf{P}^{N}\right]$ be a homogeneous ideal. Does $\rho(I)=1$ imply $I^{(r)}=I^{r}$ for all $r \geq 1$ ?

\section{REFERENCES}

[PSC] T. Bauer, S. Di Rocco, B. Harbourne, M. Kapustka, A. Knutsen, W. Syzdek, and T. Szemberg. A primer on Seshadri constants, to appear in the AMS Contemporary Mathematics series volume "Interactions of Classical and Numerical Algebraic Geometry," proceedings of a conference in honor of A.J. Sommese, held at Notre Dame, May 22-24, 2008.

[BH] C. Bocci and B. Harbourne. Comparing powers and symbolic powers of ideals, Journal of Algebraic Geometry, to appear.

[C] G. Castelnuovo. Ricerche generali sopra i sistemi lineari di curve piane, Mem. Accad. Sci. Torino, II 42 (1891).

[CM] C. Ciliberto and R. Miranda. Nagata's conjecture for a square number of points, Ricerche di Matematica 55 (2006), 71-78. MR2248163 (2007d:14098)

[ELS] L. Ein, R. Lazarsfeld and K. Smith. Uniform bounds and symbolic powers on smooth varieties, Invent. Math. 144 (2001), 241-252. MR1826369 (2002b:13001)

[E] L. Evain. Computing limit linear series with infinitesimal methods, Ann. Inst. Fourier (Grenoble) 57 (2007), 1947-1974. MR2377892(2009e:14044) 
[G] A. Gimigliano. On linear systems of plane curves, Thesis, Queen's University, Kingston, ON, Canada, 1987

[GuH] E. Guardo and B. Harbourne. Resolutions of ideals of six fat points in $\mathbf{P}^{2}$, J. Alg. 318 (2) (2007), 619-640. MR2371962(2009a:13019)

[H1] B. Harbourne. Birational models of rational surfaces, J. Alg. 190 (1997), 145-162. MR 1442149 (98a:14023)

[H2] B. Harbourne. Free resolutions of fat point ideals on $\mathbf{P}^{2}$, J. Pure Appl. Alg. 125 (1998), 213-234. MR 1600024 (99d:13016)

[H3] B. Harbourne. An algorithm for fat points on $\mathbf{P}^{2}$, Can. J. Math. 52 (2000), 123-140. MR 1745704 (2001g:13037)

[H4] B. Harbourne. The geometry of rational surfaces and Hilbert functions of points in the plane, Proceedings of the 1984 Vancouver Conference in Algebraic Geometry, CMS Conf. Proc., 6, Amer. Math. Soc., Providence, RI, 1986, pp. 95-111. MR846019 (87k:14041)

[H5] B. Harbourne. Rational surfaces with $K^{2}>0$, Proc. Amer. Math. Soc. 124 (1996), 727-733. MR1307526 (96f:14045)

[H6] B. Harbourne. Complete linear systems on rational surfaces, Trans. Amer. Math. Soc. 289 (1985), 213-226. MR779061 (86h:14030)

[H7] B. Harbourne. Global aspects of the geometry of surfaces, Ann. Univ. Paedagogicae Cracoviensis Studia Mathematica, to appear.

[Hr] R. Hartshorne. Algebraic Geometry, Graduate Texts in Mathematics, 52, Springer-Verlag, New York, 1977. MR0463157 (57:3116)

[Hi] A. Hirschowitz. Une conjecture pour la cohomologie des diviseurs sur les surfaces rationelles génériques, J. Reine Angew. Math. 397 (1989), 208-213. MR.993223 (90g:14021)

[Ho] M. Hochster. Criteria for equality of ordinary and symbolic powers of primes, Math. Z. 133 (1973), 53-65. MR0323771 (48:2127)

[HoH] M. Hochster and C. Huneke. Comparison of symbolic and ordinary powers of ideals, Invent. Math. 147 (2002), no. 2, 349-369. MR.1881923 (2002m:13002)

[HH] S. Huckaba and C. Huneke. Powers of ideals having small analytic deviation, Amer. J. Math. 114 (1992), 367-403. MR1156570 (93g:13002)

[LS] A. Li and I. Swanson. Symbolic powers of radical ideals, Rocky Mountain J. of Math. 36 (2006), 997-1009. MR2254374 (2007k:13004)

[M] H. Matsumura. Commutative Algebra. W. A. Benjamin, New York, 1970. MR0266911 (42:1813)

[Mo] S. Morey. Stability of associated primes and equality of ordinary and symbolic powers of ideals, Comm. Alg. 27(7) (1999), 3221-3231. MR.1695295 (2000e:13034)

[MNV] S. Morey, S. Noh and W. Vasconcelos. Symbolic powers, Serre conditions and CohenMacaulay Rees algebras. Manuscripta Math. 86 (1995), 113-124. MR.1314152 (96b:13002)

[R] J. Roé. Limit linear systems and applications, preprint (arXiv: math.AG/0602213.pdf)

[S] B. Segre. Alcune questioni su insiemi finiti di punti in geometria algebrica, Atti Convegno Intern. di Geom. Alg. di Torino (1961), Rattero, Turin, 1962, pp. 15-33. MR0146714 (26:4234)

[T] H. Terakawa. The d-very ampleness on a projective surface in characteristic $p$, Pac. J. Math. 187 (1999), 187-199. MR.1674325 (99m:14014)

Dipartimento di Scienze Matematiche e Informatiche "R. Magari", Università degli Studi di Siena, Pian dei mantellini, 44, 53100 Siena, Italy

E-mail address: bocci240unisi.it

Department of Mathematics, University of Nebraska, Lincoln, Nebraska 68588-0130

E-mail address: bharbour@math.unl.edu 